\title{
Derivation of a New Bioscore for Predicting Mortality in Sepsis
}

\author{
Wan Fadzlina Wan Muhd Shukeri ${ }^{1}$, Azrina Md. Ralib ${ }^{1}$, Mohd Basri Mat-Nor ${ }^{1}$ \\ ${ }^{1}$ Department of Anesthesiology and Intensive Care, Kuliyyah of Medicine, \\ International Islamic University of Malaysia
}

Presenter: Wan Fadzlina Wan Muhd Shukeri

Introduction: Currently, there is a lack of clinically feasible and reliable method for discriminating outcome in sepsis. We aimed to derive a new bioscore for predicting 30-day mortality in sepsis using a combination of biomarkers and clinical indexes. Materials and Methods: This secondary analysis of a prospectively collected data included 159 septic patients admitted to an intensive care unit (ICU). We collected data for key variables considered for inclusion in the score which included: age, sex, source of admission, comorbidities, microorganism, bacteraemia, site of infection, septic shock status, baseline Simplified Acute Physiological Score II, Sequential Organ Failure Assessment (SOFA) score (total and organ sub-scores), C-reactive protein, procalcitonin and interleukin-6 (IL-6). Approximate quintiles of each variable were assigned points based on the strength of their association with 30-day mortality. Results: Based on the statistical significance in the logistic regression model, the final score used candidate variables of age, central nervous system and liver SOFA sub-scores and IL- 6 . The bioscore predicted 30-day mortality with a very good performance [area under the receiver operating characteristic curve $0.814(95 \% \mathrm{Cl} 0.745-0.871, \mathrm{p}<0.0001)]$ in our sepsis cohort. A bioscore greater than 4 predicted 30 -day mortality with $80.4 \%$ sensitivity, 69.9\% specificity, 2.67 positive likelihood ratio and 0.28 negative likelihood ratio. As the score increased, so did mortality rate. Conclusion: A new bioscore combining age, central nervous system and liver SOFA sub-scores and IL-6 measured on ICU admission improves prediction of mortality in sepsis. 